\title{
Feasibility of in-house rapid prototyping of cardiovascular three-dimensional models for planning and training non-standard interventional procedures
}

\author{
Jarosław Meyer-Szary ${ }^{1}$, Lidia Woźniak-Mielczarek ${ }^{1}$, \\ Dominika Sabiniewicz ${ }^{2}$, Robert Sabiniewicz ${ }^{1}$ \\ ${ }^{1}$ Department of Pediatric Cardiology and Congenital Heart Diseases, Medical University of Gdansk, Poland \\ ${ }^{2} 2^{\text {nd }}$ Department of Radiology, Medical University of Gdansk, Poland
}

\section{Introduction}

Interventional treatment has become the golden standard for many congenital heart diseases and pathologies that were just recently considered unsuitable for an interventional approach are now being successfully treated by those who are most experienced. Still, being on the cutting-edge is a source of uncertainty and risk. New technologies, like rapid prototyping also know as three-dimensional (3D) printing may help to reduce this uncertainty. It has been demonstrated that 3D printed models are helpful in planning surgical and interventional treatment of various congenital heart diseases of unusual anatomy [1,2]. Less is known of application $3 \mathrm{D}$ printing for planning and rehearsing management of rare complications of previous treatments.

\section{The problem}

A 39-year-old female was treated for coarctation of the aorta with bypass-grafting from the left-subclavian to the distal descending aorta 25 years ago. Recent follow-up echocardiography revealed pressure gradient $30 \mathrm{mmHg}$ in the distal aortic arch. A computed tomography has revealed a torturous course of the aortic arch subclavian - bypass-graft route but there was no discrete narrowing. There was no continuity via the isthmic aortic segment. Additionally, there was a $5.0 \times 6.0 \mathrm{~cm}$ aortic aneurism caudally from the distal arch, most of which was filled with thrombotic masses. The active lumen was $2.5 \times 3.0 \mathrm{~cm}$ with a bottleneck-like inlet $6 \times 8 \mathrm{~mm}$ (Fig. 1A). The aneurismal mass was compressing the left pulmonary artery, significantly restricting its lumen. The pulmonary perfusion scan (SPECT-CT) has revealed no perfusion of the apical-posterior lobe, residual perfusion of the anterior lobe and diminished perfusion of the remaining left lung resulting in a total left-to-right perfusion ratio of $24 \% / 76 \%$.

Consequently, the patient was admitted to the University Clinical Center in Gdansk, GUCH Unit for treatment. The extent and approach to the treatment were considered including total resection of the aneurismal mass and reconstruction of the left pulmonary artery, but considering the asymptomatic condition of the patient a more conservative approach was preferred - interventional closure of the aneurism. Several problems needed to be addressed. Firstly, the device type and size, considering a risk of narrowing the aortic arch lumen at the site of implantation. Secondly, the route of introducing the device - the curvature of the arch and its branches, as well as the orientation of the inlet to the aneurism had to be considered. The imagery gave background on the anatomy, but the non-standard nature of the procedure made it difficult to plan the procedure with satisfying certainty.

Address for correspondence: Jarosław Meyer-Szary, MD, PhD, Assistant teacher, Department of Pediatric Cardiology and Congenital Heart Diseases, Medical University of Gdansk, ul. M. Skłodowskiej-Curie 3a, 80-210 Gdańsk, Poland, tel: +485834928 82, fax: +485834928 90, e-mail: jmeyerszary@gumed.edu.pl

Received: 28.08.2019

Accepted: 28.10.2019 


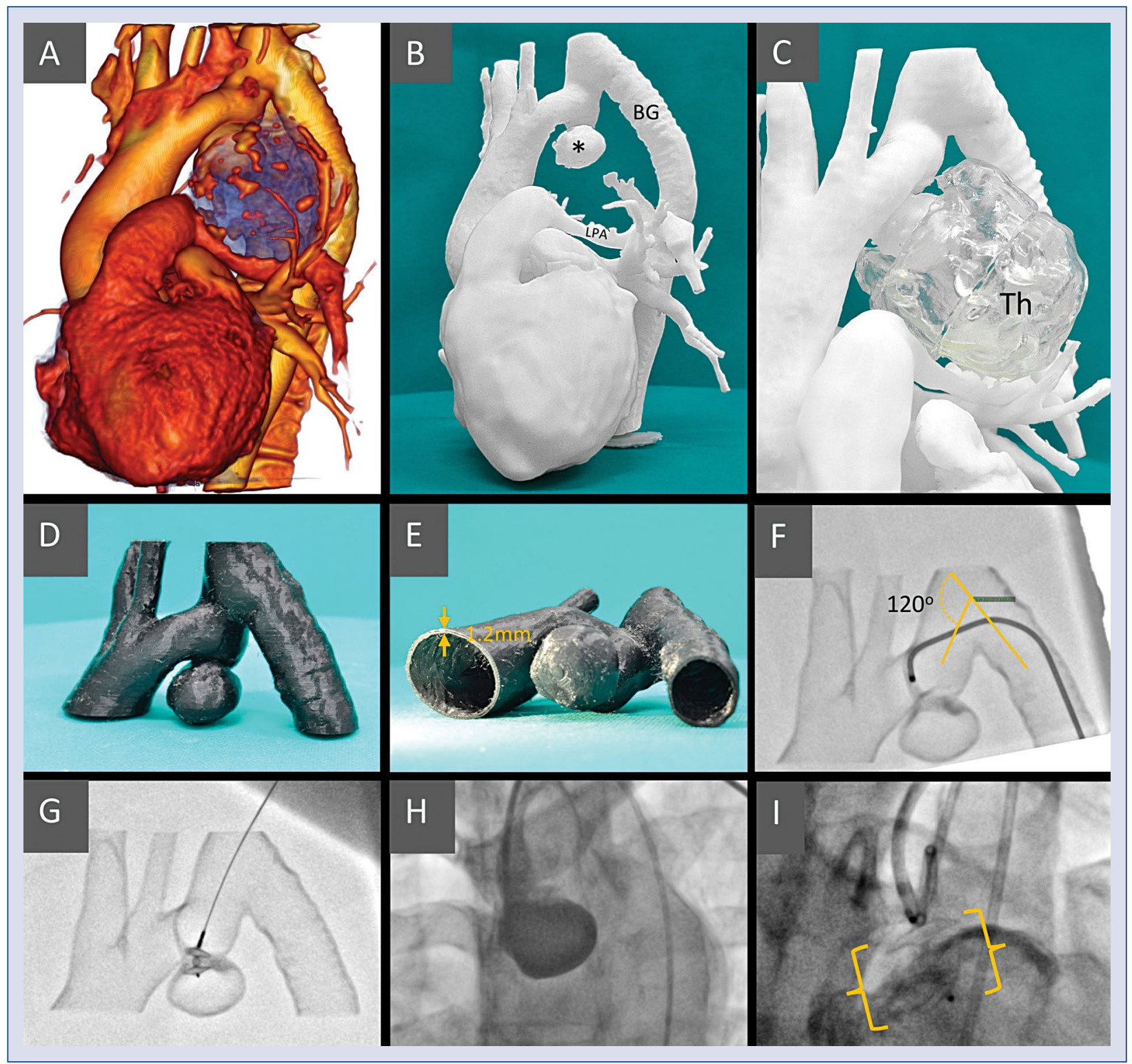

Figure 1. A. Volume rendering of the computed tomography left antero-lateral aspect; the blue area represents the thrombotic mass with numerous calcifications on the surface; B. Model I, active aneurism $\left({ }^{*}\right)$, bypass-graft (BG) and the compressed left pulmonary artery (LPA) are clearly visible; C. Model I including the thrombotic part of the aneurismal mass (Th) made separately of translucent material; D, E. Model II — region of interest, hollow vessels for simulation and training made of semi-flexible material with $1.2 \mathrm{~mm}$ wall thickness to ensure model printability and durability; F. The simulated procedure from the femoral access, it was difficult to pass through the 120 degree angle to reach the implantation site; $\mathbf{G}$. The simulated procedure from the left radial access, a straight route to the aneurism, the device is introduced to the implantation site; $\mathbf{H}$. The treatment procedure - the sheath introduced from left radial site, secondary access from femoral to inject contrast medium. ADO II at the implantation site; I. The treatment procedure - the sheath introduced from left radial site, secondary access from femoral to inject contrast medium. ADO II at the implantation site.

\section{The solution}

Two 3D printed models were made, both 1:1 scale. Model I was a whole-heart and vessels "solidified contrast” type (Fig. 1B, C). Its purpose was to depict the anatomy and spatial relations of the vessels and the aneurism. Model II, limited to the region of interest, i.e. the aneurism and the adjacent vessels, was an empty-inside (hollow) training model (Fig. 1D, E). Representing 
Table 1. Time and resources necessary to complete the models. This should be considered an example - the processing times vary, and depend on user experience and software, while the print times depend on the printer and print settings. The transparent part was done separately and was not considered crucial for the model and is not included in the cost analysis.

\begin{tabular}{|c|c|c|c|}
\hline Step & Time/resource & Model I (anatomy) & Model II (simulation) \\
\hline 1. & DICOM to STL conversion & & \\
\hline 2. & Digital model & & \\
\hline 3. & Digital model (hollow) & $\mathrm{n} / \mathrm{a}$ & $0 \mathrm{~h} 40 \mathrm{~min}$ \\
\hline 4. & Print file & $0 \mathrm{~h} 20 \mathrm{~min}$ & $0 \mathrm{~h} 20 \mathrm{~min}$ \\
\hline 5. & Printing & 2 days 13 h $34 \mathrm{~min}$ & $16 \mathrm{~h} 42 \mathrm{~min}$ \\
\hline 6. & Post-processing & $1 \mathrm{~h} 15 \mathrm{~min}$ & 0 h $25 \min (3$ h $20 \mathrm{~min})$ \\
\hline 7. & Printer & Zortrax M200 Plus & Zortrax Inveture \\
\hline 8. & Material type & Ultrat & Semi-Flex + Support Plus \\
\hline 9. & Material usage & $236 \mathrm{~g}$ & $18 g+16 g$ \\
\hline
\end{tabular}

n/a - not applicable

the vessels lumen, its purpose was to allow for a simulated procedure.

The source DICOM imagery was pre-processed in OsiriX MD software and further processed in Meshmixer to repair mesh errors and remove artefacts. At this point, preparations of the two models branched. Model I was passed on to the printer software (Z-Suite) and after applying print parameters, a printable file was generated. Model II was further processed in Meshmixer a region of interest was separated, and the model was hollowed before a printable file was generated. Both models were checked for quality and precision using a measuring tool to compare the diameters with the source imagery. The processing and print details are presented in Table 1.

Based on the imagery and the digital model, a device type and size were preliminarily selected - ADO II $6 \mathrm{~mm}$. The Model II was used to simulate the procedure. It has been confirmed that the right radial and femoral (Fig. 1F) approaches are suboptimal because of the angles on the way of the catheter and the preferred site was left radial artery (Fig. 1G). The preselected implant was possible to be implanted and had a stable position at the implantation site. Moreover, the aortic lumen did not seem to be significantly obstructed after the implantation. The procedure simulation from the preferred site was repeated 5 times. The first attempt took $3 \mathrm{~min} 20 \mathrm{~s}$, the last one $1 \mathrm{~min} 40 \mathrm{~s}$. It was clear that the time to complete was reduced owing to the learning curve of the operator.
The patient treatment was carried out ably and uneventfully, with no complications (Fig. 1H-I). According to the operator (RS), both models were accurate representations of the anatomy and the training model faithfully simulated the actual procedure. Consequently, both models contributed significantly to the success of the treatment.

\section{Conclusions}

It is possible to rapidly prototype high quality $3 \mathrm{D}$ printed models in-house, at a limited cost provided experienced staff and facilities are present. The main advantages of in-house printing are speed, and the possibility to directly oversee the process at every step to ensure the highest precision possible. Training simulators such as the one depicted above facilitate a more individualized approach to the patient - a trend currently going by the name personalized medicine.

Conflict of interest: None declared

\section{References}

1. Valverde I, Gomez G, Coserria JF, et al. 3D printed models for planning endovascular stenting in transverse aortic arch hypoplasia. Catheter Cardiovasc Interv. 2015; 85(6): 1006-1012, doi: 10.1002/ccd.25810, indexed in Pubmed: 25557983.

2. Sabiniewicz R, Meyer-Szary J, Potaż P, et al. Melody valve implantation pre-procedural planning using custom-made $3 \mathrm{D}$ printed model of the region of interest. Adv Interv Cardiol. 2018; 14(2): 210-211, doi: 10.5114/aic.2018.76419, indexed in Pubmed: 30008780. 